\title{
Phase-locked spatial domains and Bloch domain walls in type-II optical parametric oscillators
}

\author{
Gonzalo Izús* and Maxi San Miguel \\ Instituto Mediterráneo de Estudios Avanzados, IMEDEA (CSIC-UIB), ${ }^{\dagger}$ E-07071 Palma de Mallorca, Spain \\ Marco Santagiustina \\ Istituto Nazionale di Fisica della Materia, Dipartimento di Elettronica e Informatica, Università di Padova, Via Gradenigo 6/a, \\ 35131 Padova, Italy
}

(Received 15 May 2001; published 29 October 2001)

\begin{abstract}
We study the role of transverse spatial degrees of freedom in the dynamics of signal-idler phase locked states in type-II optical parametric oscillators. Phase locking stems from signal-idler polarization coupling which arises if the cavity birefringence and/or dichroism is not matched to the nonlinear crystal birefringence. Spontaneous Bloch domain wall formation is observed numerically and the dynamics and chiral properties of the fronts are investigated. Bloch walls connect homogeneous regions of self-phase-locked solutions by means of a polarization transformation. The parameter range for phase locking is found analytically. The polarization properties and the dynamics of walls in one and two transverse spatial dimensions are explained. The transition from Bloch to Ising walls is characterized, the control parameter being the linear coupling strength. The wall dynamics governs spatiotemporal dynamical states of the system, which include transient curvature driven domain growth, persistent dynamics dominated by spiraling defects for Bloch walls, and labyrinthine pattern formation for Ising walls.
\end{abstract}

DOI: 10.1103/PhysRevE.64.056231

PACS number(s): 42.65.Sf, 42.65.Yj, 42.50.-p

\section{INTRODUCTION}

Optical parametric oscillators (OPO's) are versatile nonlinear optical devices [1] with a variety of possible applications including as useful alternatives to lasers and the generation of light with nonclassical properties [2,3]. For optical cavities with large Fresnel number, they have also become a paradigm for the study of the transverse pattern formation that arises in optical systems as a consequence of diffraction and nonlinearity [4-6]. Experimental observations of such patterns have been reported [7]. Recent interest in these transverse structures in OPO's arises from the study of macroscopic manifestations of quantum phenomena in the spatial correlations present in these patterns [3], as well as from the study of spatially localized structures, such as domain walls and cavity solitons [8-15], with possible applications in alloptical signal processing.

In a type-I OPO the signal and idler fields generated in the down-conversion process have the same state of linear polarization. In a type-II OPO the signal and idler are orthogonally polarized. The additional vectorial degree of freedom of type-II OPO's is very interesting from the point of view of possible nonlinear phenomena. An interesting example of these possibilities has recently been observed experimentally and described theoretically $[16,17]$ when considering direct intracavity polarization coupling: It is possible to reach a situation of frequency degeneracy and phase locking between the orthogonally polarized signal and idler fields. This

\footnotetext{
*Permanent address: Departamento de Física, Facultad de Ciencias Exactas y Naturales, Universidad Nacional de Mar del Plata, Funes 3350 (7600) Mar del Plata, Argentina. Electronic address: izus@mdp.edu.ar.

${ }^{\dagger}$ URL: http://www.imedea.uib.es/PhysDept
}

is important because, without direct polarization coupling, a type-II OPO remains nondegenerate at frequency degeneracy because of polarization. In this phase-locked situation the polarization of the output field is determined by the locked value of the relative phase between signal and idler, which can be tuned by changes of experimentally accessible parameters. This device has been proposed as a candidate to generate bright quantum entangled states. Our general aim in this paper is to consider such phase-locked states in a cavity of large Fresnel number, and to explore how the transverse spatial degrees freedom enter in the description of the phenomenon. We find that equivalent phase-locked solutions grow locally, forming spatial domains separated by domain walls. We study the nature and dynamics of these domain walls.

When considering transverse spatial degrees of freedom in a type-II OPO without direct polarization coupling there are two different regimes. In one of them, characterized by an effective negative detuning [18], a finite wave number is selected at threshold. In the second regime, to be considered in this paper, and which occurs for the opposite sign of detuning, homogeneous solutions are selected at threshold. However, there is a continuum of possible solutions with arbitrary relative phase between signal and idler. Therefore, there are no possible domain walls. This is different from what happens in type-I OPO's in which, for the equivalent regime of detunings, homogeneous solutions with two possible opposite phases can be selected. As a consequence, spatial phase domains appear in the system separated by domain walls $[8,9,11-15,19,20]$. Such domain walls are of Ising type, that is, fronts for which the field vanishes at the core of the wall [21]. Domain walls with the same symmetry properties have also been reported for a variety of other optical systems [22-28]. Direct polarization coupling in type-II OPO's breaks the invariance under changes of relative phase, 
allowing for the formation of domain walls. These walls, however, can be of either Ising or Bloch type [10,21]. The differences between Ising and Bloch walls are that there are two equivalent Bloch ways to connect two spatial domains (symmetry breaking) and that Bloch walls can move spontaneously, leading to complicated persistent dynamical states of the system. The transition from Ising to Bloch walls is controlled by the strength of the polarization coupling. Bloch walls have recently been predicted in other optical systems [29].

Direct polarization coupling between signal and idler in type-II OPO's has been discussed in the literature $[16,17,30,31]$ by considering the insertion in the optical cavity of wave plates (such as quarter-wave or half-wave plates). In these previous studies the transverse spatial degrees of freedom were not considered. The generic phenomena described in this paper are expected for any form of direct polarization coupling. However, we address specifically the signal-idler coupling arising from birefringence and dichroism of the cavity mirrors, although our equations give a general representation of the possible forms of polarization coupling. A small amount of birefringence or dichroism is always present due to weak cavity imperfections and therefore the phenomenon considered here should be generally present in type-II OPO's.

The paper is organized as follows. Section II presents our general model equations, which are derived in detail in an Appendix. In Sec. III we calculate the OPO threshold and describe the possible stationary phase-locked homogeneous solutions, and their polarization properties are characterized in terms of the Stokes parameters. In Sec. IV we discuss domain walls in one transverse spatial dimension (1D): Ising and Bloch walls, their dynamics, and the Bloch-Ising transition are characterized. We also describe the polarization properties of these walls. Sections V and VI describe the dynamical states in two transverse spatial dimensions (2D), governed, respectively, by Bloch and Ising walls. Our main conclusions are summarized in Sec. VII.

\section{EQUATIONS FOR A TYPE-II OPO WITH DIRECT POLARIZATION COUPLING}

A type-II OPO that consists of a ring optical resonator, filled with a birefringent, nonlinear quadratic medium, will be considered. The device is externally pumped by a laser beam, uniform in the plane transverse to the cavity longitudinal axes and of frequency $\omega_{p}$. We take into account the effects of birefringence and dichroism, which can be due either to small imperfections of the cavity mirrors or to weakly birefringent (e.g., wave plates) or dichroic optical devices inserted in the optical cavity. The derivation of the governing equations is presented, for clarity, in the Appendix. For the sake of simplicity, the cavity birefringence and dichroism are supposed to be due to only one of the resonator mirrors. Note also that, in general, the mirror principal axes (i.e., those along which the Jones matrix [32] that represents the polarization transformation is diagonal) are rotated with respect to the principal axes of the crystal (i.e., those along which the susceptibility matrix is diagonal). This rotation angle $(\phi)$ is an important experimental parameter through which the strength of the effects we describe below can be controlled. We note that the equations are obtained in the mean field, paraxial, and single longitudinal mode approximation for all the fields involved.

The equations that describe the time evolution couple together four field envelopes that depend on the transverse coordinates $x, y$ : the linear polarization components of the intracavity field at the pump frequency, $B_{x, y}(x, y, t)$, and the signal and idler fields, $A_{x, y}(x, y, t)$. The signal and the idler can be either frequency degenerate or nondegenerate, depending on the frequency selection rules imposed by the combined effects of the parametric down-conversion, the cavity resonances, and the phase matching [33-35], but they are always polarization nondegenerate (type-II interaction). Hereafter the frequency degenerate (or quasidegenerate) case, which is routinely obtained by tuning the phasematching conditions [36], will be considered. Moreover, with no loss of generality we set $A_{x}$ and $B_{x}$ to be ordinary polarized beams and $A_{y}$ and $B_{y}$ to be extraordinary polarized [18]. Then the equations describing the OPO are

$$
\begin{gathered}
\partial_{t} B_{x}=\gamma_{x}^{\prime}\left[-\left(1+i \Delta_{x}^{\prime}\right) B_{x}+i \alpha_{x}^{\prime} \nabla^{2} B_{x}+c_{x}^{\prime} B_{y}\right. \\
\left.+2 i K_{0} A_{x} A_{y}+E_{0}\right], \\
\partial_{t} B_{y}=\gamma_{y}^{\prime}\left[-\left(1+i \Delta_{y}^{\prime}\right) B_{y}+i \alpha_{y}^{\prime} \nabla^{2} B_{y}+c_{y}^{\prime} B_{x}\right], \\
\partial_{t} A_{x}=\gamma_{x}\left[-\left(1+i \Delta_{x}\right) A_{x}+i \alpha_{x} \nabla^{2} A_{x}+c_{x} A_{y}+i K_{0} A_{y}^{*} B_{x}\right], \\
\partial_{t} A_{y}=\gamma_{y}\left[-\left(1+i \Delta_{y}\right) A_{y}+i \alpha_{y} \nabla^{2} A_{y}+c_{y} A_{x}+i K_{0} A_{x}^{*} B_{x}\right] .
\end{gathered}
$$

The coefficients $\gamma_{x, y}, \gamma_{x, y}^{\prime}$ (effective cavity decay rates), $\Delta_{x, y}, \Delta_{x, y}^{\prime}$ (effective cavity mode detunings), and $\alpha_{x, y}, \alpha_{x, y}^{\prime}$ (diffraction coefficients) are defined in the Appendix [see Eqs. (A14)]. Some general remarks are worth making to show differences and common features between these coefficients and those previously defined for a "perfect" cavity (see, for example, [5,18]). Due to the birefringence of the nonlinear crystal and the dichroism of the cavity, the coefficients of equivalent terms, in different equations, are slightly different, even for frequency degenerate fields. In fact they all depend on the relative refractive index and mirror transmittivity, which are polarization dependent [see Eqs. (A14) for details]. The cavity birefringence can also cause the effective detuning coefficients $\Delta_{x}, \Delta_{y}\left(\Delta_{x}^{\prime}, \Delta_{y}^{\prime}\right)$ to be different, even at frequency degeneracy. Other parameters are the nonlinearity $K_{0}$ [defined by Eq. (A15)] and the injected pump amplitude $E_{0}$ which is taken as a real number. This gives no loss of generality because it is equivalent to fixing a common phase reference for all fields. For the sake of simplicity, we take the pump to be linearly polarized in a direction parallel to the phase-matched component of the intracavity field $B_{x}$. Hence, the highly mismatched component $B_{y}$ is neither pumped nor nonlinearly coupled with the other components. It is therefore very weakly involved in the dynamics, in spite of the linear coupling with $B_{x}$. 
The linear coupling coefficients $c_{x, y}, c_{x, y}^{\prime}$ account for the dichroism and the birefringence of the cavity. They are

$$
\begin{gathered}
c_{x, y}=\frac{p+i \delta}{T \pm p \cos (2 \phi)} \sin (2 \phi), \\
c_{x, y}^{\prime}=\frac{p^{\prime}+i \delta^{\prime}}{T^{\prime} \pm p^{\prime} \cos (2 \phi)} \sin (2 \phi),
\end{gathered}
$$

where the plus (minus) sign applies for the $x(y)$ polarized component. Although their derivation and the exact relation to the physical parameters describing the cavity can be found in the Appendix it is useful to describe them briefly at this stage. The mirror dichroism is represented by the ratio between the difference of the reflectivity and the average reflectivity at a certain frequency: $2 p$ for $A_{x, y}$ (signal and idler) and $2 p^{\prime}$ for $B_{x, y}$ (pump components). The mirror birefringence causes a different phase change: $2 \delta$ for $A_{x, y}$ and $2 \delta^{\prime}$ for $B_{x, y}$. Finally, $T\left(T^{\prime}\right)$ is the average transmittivity of the signal (idler) (pump components) and $\phi$ is the relative angle of rotation between the crystal and cavity birefringence axes (the axes of dichroism are supposed to coincide with those of the cavity birefringence for the sake of simplicity). We note that similar linear coupling terms between signal and idler were previously considered $[16,17]$ by considering the insertion of wave plates (such as quarter-wave or half-wave plates) in the cavity of a type-II OPO. In these cases the general relation $c_{x}=-c_{y}^{*}$ is satisfied.

\section{THRESHOLD ANALYSIS AND HOMOGENEOUS PHASE-LOCKED SOLUTIONS}

\section{A. Threshold analysis}

The trivial solution of Eqs. (1) corresponds to the case in which a type-II OPO is below the threshold of signal generation. It is given by

$$
\begin{gathered}
A_{x}=A_{y}=0, \\
B_{x}=\tilde{c} E_{0}, \\
B_{y}=\frac{c_{y}^{\prime} \tilde{c} E_{0}}{1+i \Delta_{y}^{\prime}},
\end{gathered}
$$

where $\quad \tilde{c}=c^{r}+i c^{i}=\left(1+i \Delta_{y}^{\prime}\right) /\left[1-\Delta_{x}^{\prime} \Delta_{y}^{\prime}-c_{x}^{\prime} c_{y}^{\prime}+i\left(\Delta_{x}^{\prime}\right.\right.$ $\left.\left.+\Delta_{y}^{\prime}\right)\right]$. The threshold for instability is determined by linearizing Eqs. (1) around this solution and looking for values of the bifurcation parameter $E_{0}$ (the pump amplitude) for which perturbations grow. The general type of perturbation is given, as usual, by plane waves $\exp (i \vec{q} \cdot \vec{r}-\lambda t)$, where $\lambda(\vec{q})$ is the growth rate of the perturbation and $\vec{q}$ is its transverse wave vector. We first recall the main results of the analysis for $c_{x, y}=c_{x, y}^{\prime}=0$ [18]. In that case the trivial solution is stable for $E_{0}<E_{c}$, where

$$
E_{c}=\frac{\left(1+i \Delta_{x}^{\prime}\right)}{K_{0}} \sqrt{1+\tilde{\Delta}^{2}}
$$

and where the effective detuning $\widetilde{\Delta}$ is defined as

$$
\tilde{\Delta}=\frac{\gamma_{x} \Delta_{x}+\gamma_{y} \Delta_{y}}{\gamma_{x}+\gamma_{y}} .
$$

If the pump amplitude exceeds $E_{c}$, the steady state becomes unstable and signal and idler fields are generated. In particular, for negative effective detuning, pattern formation occurs, as studied in Ref. [18]: for this reason hereafter only the case $\tilde{\Delta}>0$ will be considered. In this case there is a Hopf bifurcation in which homogeneous perturbations $\vec{q}=\overrightarrow{0}$ with

$$
\operatorname{Im}(\lambda)=\omega=-\frac{\gamma_{x} \gamma_{y}}{\gamma_{x}+\gamma_{y}}\left(\Delta_{x}-\Delta_{y}\right)
$$

have the largest growth rate. At threshold, a family of homogeneous oscillating solutions bifurcate from the trivial steady state. For $c_{x, y}=0$, Eqs. (1) are invariant under the transformation $A_{x} \rightarrow A_{x} \exp (i \phi), A_{y} \rightarrow A_{y} \exp (-i \phi)$, and therefore the relative phase between the homogeneous oscillating solutions $A_{x}, A_{y}$ is arbitrary.

The introduction of the polarization coupling $c_{x, y} \neq 0$ breaks the invariance under changes of the relative phase. One expects that such coupling should be able to produce phase-locked homogeneous stationary (i.e., zero frequency) solutions above threshold. The linear stability analysis of Eq. (3) is rather cumbersome when $c_{x, y} \neq 0$, and simple analytical expressions for the threshold analysis are not found. The linear stability of the trivial solution was investigated numerically and a decrease of the threshold is observed when the polarization coupling is included. As there is no closed expression for the eigenvalues, it is in this case more convenient to determine a threshold through the condition of existence of the relative phase-locked solutions. Numerical and analytical results are coincident. We consider the special case $c_{x}=c_{y}=c_{x}^{r}+i c_{x}^{i}\left[\left(c_{x}^{r}, c_{x}^{i}\right] \in \mathcal{R}\right)$ which through Eqs. (2) can be seen to correspond to setting either $\phi= \pm \pi / 4$ or $\phi$ $= \pm 3 \pi / 4$ (i.e., to fixing the angle to the value that maximizes the coupling strength) or to setting $p=c_{x}^{r}=0$ (i.e., no dichroism). We note that setting $p=0$ reproduces a particular case of the polarization coupling considered in $[16,17]$.

Let us call $\bar{A}_{x}=a_{x} \exp \left(i \psi_{x}\right)$ and $\bar{A}_{y}=a_{y} \exp \left(i \psi_{y}\right)$ the homogeneous stationary solutions; by substituting such formulas into Eqs. (1) one gets

$$
\begin{gathered}
\sin \left(\psi_{y}-\psi_{x}\right)=\frac{\Delta_{x}-\Delta_{y}}{2 \sqrt{\left(c_{x}^{r}+c_{x}^{i} \Delta_{x}\right)\left(c_{x}^{r}+c_{x}^{i} \Delta_{y}\right)}}, \\
\cos \left(\psi_{y}+\psi_{x}\right)=\frac{1}{2 K_{0} E_{0}|\tilde{c}|^{2} \sqrt{\Gamma}}\left[\left(\Delta_{x}+\Gamma \Delta_{y}\right) c^{r}-(1+\Gamma) c^{i}\right. \\
\left.-2 \sqrt{\Gamma} \cos \left(\psi_{y}-\psi_{x}\right)\left(c_{x}^{i} c^{r}-c_{x}^{r} c^{i}\right)\right], \\
a_{x}^{2}=\frac{1}{4 K_{0}^{2} c^{r} \Gamma}\left[2 K_{0} E_{0} \sqrt{\Gamma}\left(c^{r} \sin \left(\psi_{y}+\psi_{x}\right)-c^{i} \cos \left(\psi_{y}+\psi_{x}\right)\right)\right. \\
\left.+2 \sqrt{\Gamma} c_{x}^{r} \cos \left(\psi_{y}-\psi_{x}\right)-(1+\Gamma)\right],
\end{gathered}
$$




$$
a_{y}^{2}=\Gamma a_{x}^{2}
$$

where $\Gamma=\left(c_{x}^{r}+c_{x}^{i} \Delta_{x}\right) /\left(c_{x}^{r}+c_{x}^{i} \Delta_{y}\right)$.

From Eqs. (7) we find two conditions for the existence of phase-locked homogeneous stationary solutions. The first one comes from the fact that the phase difference among the solutions [see the first of Eqs. (7)] is real only if the modulus of the right hand side is less than 1 , that is, if

$$
\left(\Delta_{y}-\Delta_{x}\right)^{2} \leqslant 4\left(c_{x}^{r}+c_{x}^{i} \Delta_{x}\right)\left(c_{x}^{r}+c_{x}^{i} \Delta_{y}\right) .
$$

This boundary in the complex plane $c_{x}^{r}, c_{x}^{i}$ defines the limit of the locking regime. Inside the boundary stationary solutions do not exist. Physically speaking, the locking condition means that stationary phase-locked solutions exist whenever the direct polarization coupling breaking is large compared with the difference in detunings. When the condition (8) is not satisfied, numerical solutions show that there are still homogeneous states but their phase varies periodically with time. Such solutions indicate the persistence of the Hopf bifurcation found for $c_{x, y}=0$ when the polarization coupling is small.

The second condition for the existence of solutions refers to the pump value above which there is signal and idler generation. The threshold $E_{c}$ can be determined by setting $a_{x}=0$ into Eqs. (7) and solving for $E_{0}$. The final result is

$$
\begin{aligned}
E_{c}^{2}= & \frac{1}{4 K_{0}^{2} \Gamma|\tilde{c}|^{2}}\left\{(1+\Gamma)^{2}+\left(\Delta_{x}+\Gamma \Delta_{y}\right)^{2}+4 \Gamma\left|c_{x}\right|^{2} \cos ^{2}\left(\psi_{y}\right.\right. \\
& \left.\left.-\psi_{x}\right)-4 \sqrt{\Gamma} \cos \left(\psi_{y}-\psi_{x}\right)\left[c_{x}^{r}(1+\Gamma)+c_{x}^{i}\left(\Delta_{x}+\Gamma \Delta_{y}\right)\right]\right\} .
\end{aligned}
$$

The classification of the solutions found above this threshold is easier to understand by considering the case $\Delta_{x}=\Delta_{y}$ for which the condition (8) is automatically satisfied. In this case and regardless of the value of $c_{x, y}$ the relative phase shift between phase-locked signal and idler can be either 0 (inphase solution) or $\pi$ (out-of-phase solution) and the amplitude of the fields is equal $\left(a_{x}=a_{y}\right)$ since $\Gamma=1$. Once the phase shift $\psi_{y}-\psi_{x}$ is known it can be substituted into the second of Eqs. (7) which can be solved for $\psi_{y}+\psi_{x}$. In principle two solutions exist for each phase difference (in- and out-of-phase cases), due to the fact that the arccos is a multivalued function in the range $[-\pi, \pi]$. However, if the negative solutions for $\psi_{y}+\psi_{x}$ are replaced in the third of Eqs. (7) the result is $a_{x}^{2}<0 \forall E_{0}$. Therefore only positive solutions of the angle sum should be taken to guarantee that $a_{x}^{2}>0$ above a certain threshold $E_{c}$. By substituting the phase difference and sum into the third equation the amplitude is finally found. There are two equivalent possible solutions for the in-phase case and two for the out-of-phase case. The existence of these two equivalent solutions is a consequence of the symmetry $\left(A_{x}, A_{y}\right) \rightarrow-\left(A_{x}, A_{y}\right)$ of Eqs. (1) which is preserved for $c_{x, y} \neq 0$. In summary, we find inphase solutions $\left(A_{x}=A_{y}\right)$ and out-of-phase solutions $\left(A_{x}=\right.$

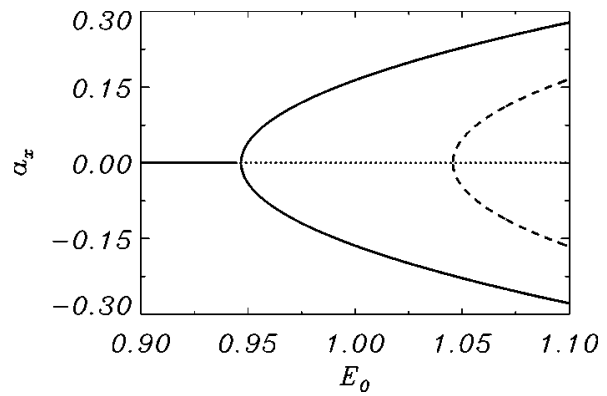

FIG. 1. Bifurcation diagram of the homogeneous solutions (solid curve stable, dashed curve unstable). Dotted line is the trivial unstable solution amplitude. Parameters are $K_{0}=1, c_{y}=c_{x}$ $=0.05(1+i), c_{x}^{\prime}=c_{y}^{\prime}=0.1, \Delta_{x}^{\prime}=\Delta_{y}^{\prime}=0, \Delta_{x}=0.01, \Delta_{y}=0.03$.

$\left.-A_{y}\right)$. Each of the in- and out-of-phase cases includes two equivalent solutions which we denote as the + and - solutions satisfying $A_{x, y}^{+}=-A_{x, y}^{-}$.

In the general case with $\Delta_{x} \neq \Delta_{y}$ solutions are no longer strictly in or out of phase. Nonetheless, well within the phase-locked regime where the detuning coefficients are small compared with the strength of the polarization coupling, the solutions one finds are close to being in or out of phase. Therefore we will still use in this situation the names of in- and out-of-phase solutions even if this is not generally a rigorous description. Other situations can occur close to the limit of the locking regime fixed by Eq. (8). For example, for $c_{x}=c_{y}$ and a purely dichroic mirror, $c_{x}^{i}=0$ so that $\Gamma=1$ and signal and idler have the same amplitude $a_{x}=a_{y}$. At the onset of the locking regime $\left|\Delta_{x}-\Delta_{y}\right|=2\left|c_{x}^{r}\right|$, and it follows from the first of Eqs. (7) that the two fields are locked at a phase difference $\psi_{y}-\psi_{x}= \pm \pi / 2$. In any case, for each locked value of the phase difference there are two equivalent + and - solutions satisfying $A_{x, y}^{+}=-A_{x, y}^{-}$

A bifurcation diagram for the homogeneous solutions for a generic case with $\Delta_{x} \neq \Delta_{y}$ is presented in Fig. 1 . The selection of the solution that actually bifurcates, either in phase or out of phase, is determined by the relative value of the threshold $E_{c}$ for each solution. Equation (9) shows that the threshold is lower if the term proportional to $\cos \left(\psi_{y}-\psi_{x}\right)$ is positive; for the in-phase solution this occurs if $c_{x}^{r}>0, c_{x}^{i}$ $>0$ and vice versa for the out-of-phase solution if $c_{x}^{r}<0, c_{x}^{i}$ $<0$. An example of the thresholds calculated for the in-phase and out-of-phase solutions, as a function of $c_{x}^{r}=c_{x}^{i}$, and for the same detuning values as in Fig. 1, is shown in Fig. 2. Note that there is a range of values of the coupling $c_{x, y}$ in which Eq. (8) is not satisfied and there is no threshold for the emergence of the phase-locked solutions. But, in the range in which Eq. (8) holds, the lower threshold decreases as the coupling strength $\left|c_{x, y}\right|$ increases, allowing parametric downconversion for lower values of the external pump with respect to the reference case $\left(c_{x, y}=0\right)$. Note also that the roles of the two solutions are exchanged if the sign of $c_{x, y}$ is reversed. Only the solutions with lower threshold are stable. From numerical investigation we learn that even in the nonextended (dynamical) system the solutions with higher threshold are unstable. We finally note that our numerical integrations show that, when switching on the pump to a 


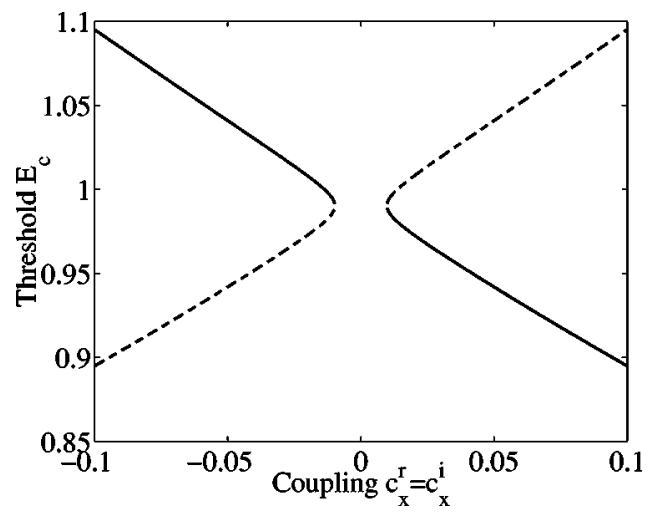

FIG. 2. Threshold of instability $E_{c}$ for the trivial stationary solution [Eq. (3)]: solid (dashed) curve is the threshold of the in(out-of-)phase solution. Here $c_{y}=c_{x}$ and the other parameters are the same as in Fig. 1.

value for which both the in-phase and out-of-phase solutions exist, the solution that is always selected is the one with lowest threshold. Therefore, in practice we find only the two equivalent solutions of lowest threshold.

\section{B. Polarization properties of the phase-locked solutions}

An important question is the polarization state of the phase-locked homogeneous stationary solutions that we have just described. It is useful to consider the polarization representation given by the normalized Stokes parameters, defined as [32]

$$
\begin{gathered}
s_{1}=\frac{\left|A_{x}\right|^{2}-\left|A_{y}\right|^{2}}{\left|A_{x}\right|^{2}+\left|A_{y}\right|^{2}}, \\
s_{2}=\frac{A_{x} A_{y}^{*}+A_{x}^{*} A_{y}}{\left|A_{x}\right|^{2}+\left|A_{y}\right|^{2}}, \\
s_{3}=\frac{-i\left(A_{x} A_{y}^{*}-A_{x}^{*} A_{y}\right)}{\left|A_{x}\right|^{2}+\left|A_{y}\right|^{2}} .
\end{gathered}
$$

These real parameters are sufficient to characterize any state of polarization of a monochromatic field by assigning to the field a point in the Poincaré sphere. The equator of the sphere $\left(s_{3}=0\right)$ corresponds to linearly polarized states and the poles of the sphere $\left(s_{1}=s_{2}=0, s_{3}= \pm 1\right)$ correspond to states of opposite circular polarization. By replacing the homogeneous solutions in Eqs. (10), the state of polarization of our phase-locked solutions is represented by

$$
\begin{gathered}
\bar{s}_{1}=\frac{1-\Gamma}{1+\Gamma}, \\
\bar{s}_{2}=\frac{2 \sqrt{\Gamma}}{1+\Gamma} \cos \left(\psi_{y}-\psi_{x}\right), \\
\bar{s}_{3}=-\frac{2 \sqrt{\Gamma}}{1+\Gamma} \sin \left(\psi_{y}-\psi_{x}\right) .
\end{gathered}
$$

These equations show that the polarization state of the optical field is determined by the locked value of the phase difference between signal and idler. For $\Delta_{x}=\Delta_{y}, \Gamma=1$ and $\sin \left(\psi_{y}-\psi_{x}\right)=0$ so that $\left(\bar{s}_{1}, \bar{s}_{2}, \bar{s}_{3}\right)=(0, \pm 1,0)$, where the plus (minus) sign applies for the in- (out-of-)phase solution. This means that the two possible phase-locked solutions are actually linear and orthogonal polarizations whose azimuth angles are $\theta=a \tan \left(\bar{s}_{2} / \bar{s}_{1}\right) / 2= \pm \pi / 4$. We mentioned before that there are two equivalent solutions for each of the in- and out-of-phase solutions. These correspond to linearly polarized states along the same direction, but in opposite senses, and they have the same Stokes parameters. The Stokes parameters are determined by the relative phase, while the two equivalent solutions have a different global phase. For example, in the two equivalent in-phase solutions $\bar{s}_{2}=1$ and $\operatorname{Re}\left(A_{x}\right)=\operatorname{Re}\left(A_{y}\right)$, but in one of the solutions $\operatorname{Re}\left(A_{x}\right)>0$ and in the other one $\operatorname{Re}\left(A_{x}\right)<0$.

When the detunings are different, the homogeneous solutions become elliptically polarized beams $\left(\bar{s}_{3} \neq 0\right)$. However, if the detunings are small $\left(c_{x}^{i} \Delta_{x, y} \ll c_{x}^{r}\right) \Gamma \simeq 1$ still and is well within the locking regime $\sin \left(\psi_{y}-\psi_{x}\right) \simeq 0$. In these circumstances the state of the beam is close to being linearly polarized $\left(\bar{s}_{3} \simeq 0\right)$ with azimuth angles close to $\pm \pi / 4$. However, it is important to note that by changing the detuning parameters and the strength of the polarization coupling it is possible to explore arbitrary states of polarization. These states are determined from Eq. (11) in terms of the phase difference of the locked state. For example, in the case mentioned above of a purely dichroic mirror, $c_{x}^{i}=0$, we have $\Gamma=1$ and therefore $\bar{s}_{1}=0$. In this case, and at the onset of the locking regime, $\bar{s}_{2}=0, \bar{s}_{3}= \pm 1$ so that the locked solution is circularly polarized. Going into the locking regime, the polarization state will evolve toward linearly polarized states but keeping $\bar{s}_{1}=0$.

The threshold decrease due to the polarization coupling discussed above has now a simple physical interpretation. Let us recall that the condition $c_{x}=c_{y}$ means that the relative angle of inclination $\phi$ must be one of these values: $[ \pm \pi / 4$, $\pm 3 \pi / 4]$. In particular, through Eqs. (2), $c_{x}^{r}\left(c_{x}^{i}\right)$ is positive for $p>0(\delta>0)$ and $\phi=\pi / 4,-3 \pi / 4$ or $p<0 \quad(\delta<0)$ and $\phi=-\pi / 4,3 \pi / 4$. When $A_{x}$ and $A_{y}$ are in phase, the total field is linearly polarized at $\pi / 4$ (or $3 \pi / 4$ ) rad with respect to the crystal axes. The conclusion that can be drawn is that the intracavity field is actually oriented along one of the principal axes of the cavity birefringence dichroism. The same occurs for the out-of-phase solution which is a beam linearly polarized at an angle $-\pi / 4$ (or $3 \pi / 4$ ) rad and thus the role of the coefficients is exchanged. So the polarization selected is always aligned with one of the cavity principal axes.

\section{PHASE POLARIZATION DOMAIN WALLS IN ONE TRANSVERSE DIMENSION: BLOCH-ISING TRANSITION}

In the previous section we discussed the existence of two equivalent homogeneous solutions which we named the + and - solutions. These are the solutions with lowest thresh- 
old, while we mentioned that other solutions of higher threshold are seen to be unstable. When the OPO switches on after setting the pump to a value above its threshold value, given by Eq. (9), either the + or - solution can be selected since they have the same growth rate. When the transverse spatial degrees of freedom are taken into account, this selection, or spontaneous symmetry breaking of the homogeneous solution, can be local, with a different solution emerging in different spatial regions. It is then expected to find domain walls that separate the spatial domains with different but equivalent solutions. For either the signal or idler what changes when going from one solution to the other is just the sign. For example, $A_{x}$ takes values $\widetilde{A}$ and $-\widetilde{A}$ at opposite sides of the wall. Therefore the walls can be considered as phase walls of a complex field like the ones described for type-I degenerate OPO's $[11,13,15]$. When considering the signal and idler the domain wall separates two solutions with polarization properties determined by the locking of the relative phase of the two fields. One might then talk about polarization walls. However, we have already mentioned that the + and - solutions have the same Stokes parameters, but there is a change in the global phase of the polarization state. In this sense we refer to these walls as phase polarization walls. In any case, the polarization state might present interesting features in the core of the wall.

Phase domain walls for a complex field can be of Ising or Bloch type [10,21]. As a general characterization, in an Ising wall there is a single field profile connecting one homogeneous solution with a second equivalent one, while we talk of a Bloch type wall when there are two different field profiles (walls) connecting the two solutions. A Bloch wall implies, therefore, spontaneous symmetry breaking for the domain wall. In the following we study Ising and Bloch domain walls, the transition between them, and the polarization properties in one transverse spatial dimension. The characterization of some properties of these walls is much more clear in one dimension. Other features associated with twodimensional phenomena are postponed to the following sections.

Numerical integration [37] of Eqs. (1) confirms that stationary uniform domains of the + or - solutions form spontaneously starting from a randomly and weakly perturbed trivial steady state (3). Well within the locking regime, the domain walls are of the Ising type, but on changing the values of $c_{x, y}$ and moving toward the boundary of the locking regime we find a transition from Ising to Bloch domain walls [10]. An example of an Ising wall (IW) is presented in Fig. $3(\mathrm{a})$. It connects the + solution at $x \rightarrow-\infty$ with the + solution at $x \rightarrow \infty$. By plotting the numerically obtained solution in the complex plane $\left[\operatorname{Re}\left(A_{x}\right), \operatorname{Im}\left(A_{x}\right)\right][$ Fig. 3(b)] it is clear that the IW is characterized by a zero crossing of the field. An example of a 1D optical Bloch wall (BW) is instead given in Figs. 4(a) and 4(b); note that the field amplitude (represented by the vector modulus in the complex plane) never goes to zero and the wall consists of an almost pure phase rotation of $\pi \mathrm{rad}$. The phase can rotate in two possible senses along the interface, clockwise or counterclockwise in the complex plane. This characteristic is usually called the wall chirality and it is defined to be positive for clockwise
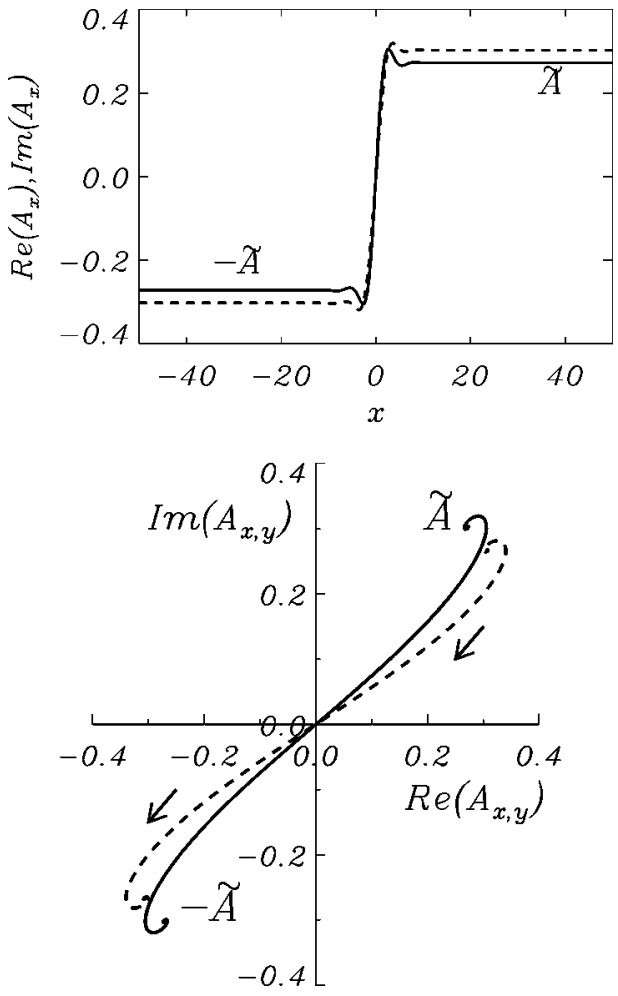

FIG. 3. Numerical solution of Eqs. (1) in one spatial dimension showing an example of an Ising wall: (a) Solid (dashed) curve is the real (imaginary) part of the field $A_{x}$ as a function of the transverse coordinate; (b) the same wall is represented in the complex plane $\left[\operatorname{Re}\left(A_{x}\right), \operatorname{Im}\left(A_{x}\right)\right]$ as a solid line. Dashed curve is the Ising wall associated with the field $A_{y}$. The parameters are $\gamma_{y}=\gamma_{y}^{\prime}=1, \gamma_{x}$ $=\gamma_{x}^{\prime}=1.001, \Delta_{x}^{\prime}=\Delta_{y}^{\prime}=0, \Delta_{x}=0.01, \Delta_{y}=0.03, \alpha_{x}^{\prime}=\alpha_{y}^{\prime}=0.125$, $\alpha_{x}=\alpha_{y}=0.25, K_{0}=1, E_{0}=1.25, c_{x}^{\prime}=c_{y}^{\prime}=0.01(1+i)$, and $c_{x}=c_{y}$ $=0.082$.

rotation, or negative for counterclockwise rotation. Therefore, there exist two equivalent domain walls of opposite chirality for $A_{x}$. One of the two appears by spontaneous symmetry breaking. In the example of Fig. 4 the wall of negative chirality for $A_{x}$ is selected.

In the example of the Ising wall of Fig. 3 the parameters are such that we are well within the locking regime and the domain wall connects homogeneous equivalent in-phase solutions in which the locked phase difference is close to zero $\left(\psi_{y}-\psi_{x} \simeq-0.122\right)$. The shape of the field $A_{y}$ across the domain wall is similar to the one of $A_{x}$. The situation is different for the example of the Bloch wall in Fig. 4. The parameters correspond here to a situation close to the boundary of phase locking and the homogeneous + and - solutions have a locked phase difference $\psi_{y}-\psi_{x}=\pi / 2$. In addition, Bloch walls for this system are characterized by the fact that the wall profile for the field $A_{y}$ has always opposite chirality to that of $A_{x}$, as seen in the example of Fig. 4(b).

The polarization characteristics of Ising and Bloch domain walls are very different. For an Ising wall the Stokes parameters are seen to remain constant across the core of the wall. This is due to the fact that the phase difference $\psi_{y}$ $-\psi_{x}$ remains fixed to its locked value while going from the - to the + solution across the wall. On the contrary, in a 

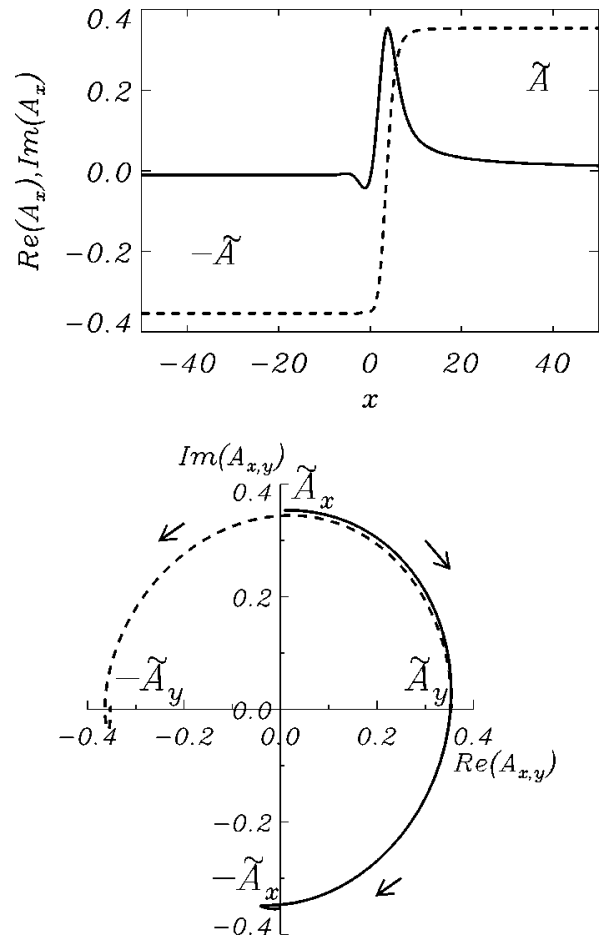

FIG. 4. Numerical solution of Eqs. (1) in one spatial dimension, showing a Bloch wall. (a) Solid (dashed) curve is the real (imaginary) part of the field $A_{x}$ as a function of the transverse coordinate; (b) the same wall is represented in the complex plane $\left[\operatorname{Re}\left(A_{x}\right), \operatorname{Im}\left(A_{x}\right)\right]$ as a solid line. Dashed curve is the Bloch wall associated with the field $A_{y}$. The parameters are the same as in Fig. 3 , except $c_{x}=c_{y}=0.01$.

Bloch wall the locked value of the phase difference $\psi_{y}-\psi_{x}$ is a function of the position while moving from one side of the wall to the other. The consequence is that the Stokes parameters have a nontrivial space dependence across the wall determining peculiar polarization characteristics of the core of the wall. As examples of such polarization characteristics we show in Fig. 5 the variation of the Stokes parameters across two examples of Bloch domain walls. In Fig. 5(a) we consider a Bloch wall that connects two linearly polarized states. In Fig. 5(b), which corresponds to the wall of Fig. 4, the wall connects two elliptically polarized states which are close to being circularly polarized.

In Fig. 5(a) the two asymptotic states for $x \rightarrow \pm \infty$ are in-phase solutions characterized by $\left(\bar{s}_{1}, \bar{s}_{2}, \bar{s}_{3}\right)=(0,1,0)$ corresponding to a linearly polarized state of azimuth $\theta=\pi / 4$. At the core of the wall $\left(\bar{s}_{1}, \bar{s}_{2}, \bar{s}_{3}\right)=(0,-1,0)$ which corresponds to a state of orthogonal linear polarization. Note that as the phase solution rotates by $\pi$ rad the Stokes parameters $s_{1}, s_{2}, s_{3}$ return to the initial values, i.e., the polarization of the total field is the same on each side of the wall, as previously mentioned. Along the wall the polarization changes, the field becoming elliptically polarized, but not in an arbitrary manner. The transformation is forced to occur for $s_{1}$ $\simeq 0$, i.e., the azimuth angle of the ellipse is fixed in practice to $\theta= \pm \pi / 4$. For $x<0$, and close to the core of the wall, $s_{3} \simeq-1$, indicating a state close to being left circularly polarized, while for $x>0$, and close to the core of the wall,
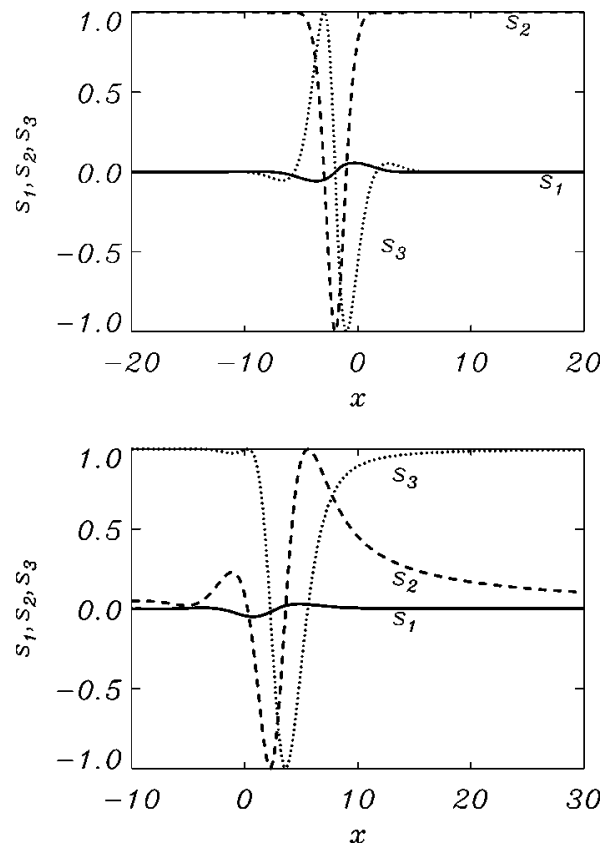

FIG. 5. Variation of the polarization along a Bloch wall, represented by means of the Stokes parameters (10) as they result from a numerical solution: $s_{1}(x)$ (solid curve), $s_{2}(x)$ (dashed), $s_{3}(x)$ (dotted). (a) The Bloch wall separates linearly polarized states. Parameters are the same as in Fig. 3, except $\gamma_{x}=\gamma_{x}^{\prime}=1, \Delta_{x}=\Delta_{y}=0.03$, $c_{x}^{\prime}=c_{y}^{\prime}=0.01, c_{x}=c_{y}=0.02$. (b) The same representation for the Bloch wall of Fig. 4, which separates elliptically polarized states.

$s_{3} \simeq 1$, indicating a state close to being right circularly polarized. In terms of the Poincare sphere, the representative point moves from a point at the equator through the vicinity of the south pole to the opposite point at the equator, and back to the original point through the vicinity of the north pole. The change of ellipticity across the wall, $\eta(x)=\arcsin \left(s_{3}\right) / 2$, yields a natural interpretation of the chirality: for a BW of negative chirality, like the one shown here, the ellipticity of the polarization state first decreases to a maximum negative value, going, as we move to the other side of the wall, to a maximum positive value. For a BW of positive chirality the excursion in ellipticity goes in the opposite direction.

In Fig. 5(b) the variation of the Stokes parameters indicates a sequence of elliptically polarized states with two points in the core of the wall at which the state becomes linearly polarized $\left(s_{3}=0\right)$. The change in polarization state still occurs for $s_{1} \simeq 0$ because, since $c_{x}^{i}=0$, still $\Gamma=1$. The representative point moves now in the Poincare sphere from a point close to the north pole, to the vicinity of the south pole, crossing the equator, and back to the original point along the other side of the sphere, again crossing the equator. An opposite sense of motion along the sphere would correspond to an opposite chirality of the wall.

We note that a quantitative precise description of the variation of the Stokes parameters can generally be given by invoking the relation, which follows from symmetry considerations, $A_{x} \simeq i A_{y}^{*}$ pointwise along the wall. The Stokes parameters as functions of the spatial variation of the phase 


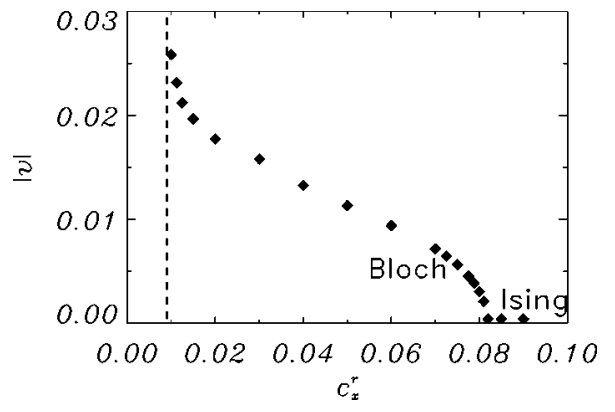

FIG. 6. Velocity of a BW as a function of $c_{x}^{r}\left(c_{x}^{i}=0\right)$ as given by numerical simulations of Eqs. (1) in the regime $\gamma_{x} \Delta_{x} \neq \gamma_{y} \Delta_{y}$. The parameters are the same as in Fig. 3, except the values of $c_{y}^{r}$ $=c_{x}^{r}$. For the last three points in the figure (corresponding to Ising walls) the velocity is zero. The dotted line indicates the onset of the locking regime.

$\psi_{x}(x)$ are then given by [substitute $A_{x}=i A_{y}^{*}$ into Eqs. (10)]:

$$
\begin{gathered}
s_{1}(x) \simeq 0, \\
s_{2}(x) \simeq \sin \left[2 \psi_{x}(x)\right], \\
s_{3}(x) \simeq-\cos \left[2 \psi_{x}(x)\right] .
\end{gathered}
$$

We next turn to considering the dynamics of the domain walls, which is also useful to determine the transition between Ising and Bloch walls. Isolated Ising walls in 1D are stable and remain stationary. The dynamics of 1D BW's depends critically on the values of the cavity decay rates and the detuning. For $\gamma_{x} \Delta_{x}=\gamma_{y} \Delta_{y}, 1 \mathrm{D}$ BW's do not move: they are stable stationary interfaces between equivalent uniform domains. A similar situation takes place in the potential limit of the 1D parametrically forced complex Ginzburg-Landau equation (PCGLE) where stationary BW's have been found analytically [21,38]. On the contrary, for $\gamma_{x} \Delta_{x} \neq \gamma_{y} \Delta_{y}$ walls of different chirality move in opposite directions, as also happens outside the potential limit of the PCGLE. The velocity of the resulting BW depends on the value of the parameters; in particular, it depends strongly on $c_{x, y}$. In Fig. 6 the velocity of BW's as a function of $c_{x}$ (real), for selected values of the other parameters, is shown as it results from numerical solutions. For small $c_{x}$ BW's are not stable because we are outside the locking regime [Eq. (8) is not satisfied]; for larger values the velocity decreases on increasing $c_{x}$ and finally it vanishes. The vanishing of the velocity identifies the transition point at which a BW decays into an IW. This transition is continuous, i.e., the amplitude of the wall solutions, which is almost constant for BW's for small $c_{x}$, shows larger and larger variations as the transition is approached. The amplitude of the signal field (and also the idler) becomes small at the core of the wall and it eventually reaches zero. At this point BW's and IW's exchange their stability and only standing IW's are found beyond that critical value. The conclusion is that in the regime of the parameter space for which BW's are stable IW's are unstable, and vice versa.

In addition to the transition from Bloch to Ising type, the strength of the direct polarization coupling coefficients $c_{x, y}$
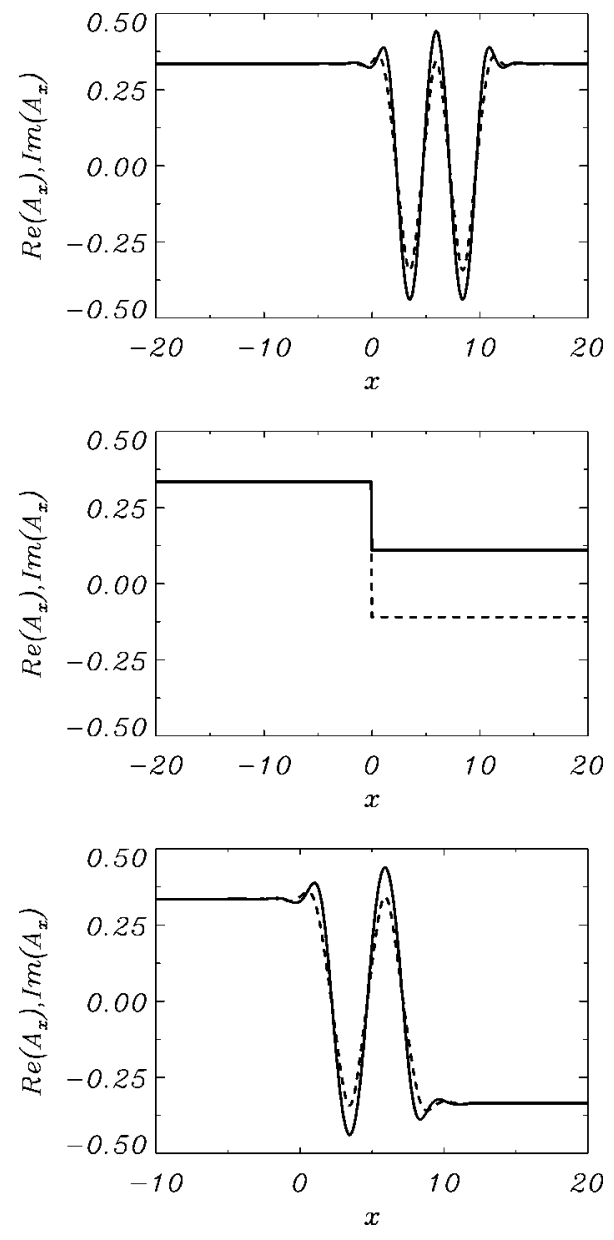

FIG. 7. Examples of multiple hump stable solutions. Solid (dashed) curve is the real (imaginary) part of the field $A_{x}$. In (a) the field comes back to the initial state; (b) initial condition used in (a). Here $\operatorname{Re}\left(A_{x}\right)=\operatorname{Im}\left(A_{x}\right)$ for $x<0$. (c) IW with two crossings of the zero amplitude point. Parameters are $\alpha_{x}=0.25375, \alpha_{y}=0.24625$ $\alpha_{x}^{\prime}=\alpha_{y}^{\prime}=0.125, \quad \Delta_{x}^{\prime}=\Delta_{y}^{\prime}=0, \quad \Delta_{x}=\Delta_{y}=0.02, \quad \gamma_{x}^{\prime}=\gamma_{y}^{\prime}=1, \quad \gamma_{x}$ $=0.985, \gamma_{y}=1.015, c_{x}^{\prime}=c_{y}^{\prime}=0.01, c_{x}=c_{y}=0.2+i 0.02$.

also controls the wall width. Moving well within the locking regime the width becomes small, while it diverges as $c_{x, y}$ $\rightarrow 0$.

We finally mention that other forms of stable localized structures or domain walls are sustained in this system, although they do not appear spontaneously when starting from a weakly perturbed trivial steady state (3) and a pump value above threshold. They can be formed far above threshold, mediated by the unstable phase-locked solutions of higher threshold discussed in Sec. III. A first example, shown in Fig. 7(a) is a stable 1D localized structure in the background of a + in-phase solution. This structure is generated from a steplike initial condition, shown in Fig. 7(b), which connects the stable + in-phase solution with the unstable + out-ofphase solution. The dynamical evolution of this unstable configuration leads to the localized structure. A second example shown in Fig. 7(c) is a kind of domain wall that connects, as in the Ising and Bloch walls discussed above, the equivalent + and - solutions of the phase-locked solutions of lowest threshold. The difference from our previous Ising 
(a)

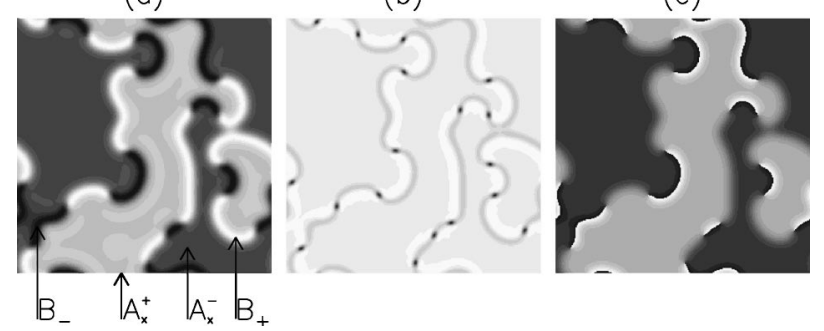

FIG. 8. A snapshot at time $t=1600$ of the field $A_{x}(x, y, t)$, spontaneously generated from random initial conditions close to the trivial steady state. (a), (b), and (c) show, respectively, the real part, the intensity, and the phase. Black and white segments in the walls $\left(B_{ \pm}\right)$in (a) correspond to opposite senses of rotation of the phase (chirality). The black points in (b) are the defects, where signal and idler amplitude vanishes. The phase varies between $-\pi$ (black) and $\pi$ (white). Intermediate values are showed in a linear scale of 256 gray levels. The parameters are the same as in Fig. 7, except $\Delta_{x}$ $=0.01, \quad \Delta_{y}=0.03, \quad c_{x}^{\prime}=c_{y}^{\prime}=0.025(1-i / 2)$, and $c_{x}=c_{y}=0.02(1$ $+i)$.

walls consists in a more complicated structure of the core of the wall. In this case the field $A_{x}$ vanishes at three points in the core of the wall. This type of domain wall emerges dynamically from a similar initial condition to the one shown in Fig. 7(b), but with a different sign of the unstable solution of the step: The initial steplike condition connects the stable + in-phase solution with the unstable - out-of-phase solution.

\section{CHARACTERIZATION OF 2D BLOCH WALLS}

In our 2D numerical solutions [37] with random initial conditions around the trivial unstable solution we also observe the spontaneous formation of BW's which are now lines in the transverse plane. These walls evolve dynamically as described below. An additional feature of BW's in 2D is that the domain walls can emerge with opposite chirality in different spatial regions along the wall. The points on the wall where the chirality changes sign are singular points: At these points the phases of the signal and idler fields are not defined and the amplitudes go to zero, i.e., they can be classified as topological defects. The BW, at that particular point, actually degenerates into an IW. A snapshot of typical transient transverse patterns generated by the Bloch walls is shown in Fig. 8 for the component $A_{x}$. The two equivalent + and - phase-locked homogeneous solutions are represented in Fig. 8(a) by regions of different intensity on a gray scale $\left(A_{x}^{ \pm}\right)$. Likewise, the segments along the walls of different chirality are represented, respectively, by black or white segments $\left(B_{ \pm}\right)$in Fig. 8(a). The defects where the changes of chirality take place can be observed as black dots in the intensity field [Fig. 8(b)]. Note that, except for the defects, the field intensity is almost constant in the domains and only slightly modulated close to the core of the wall. The phase field, shown in Fig. 8(c), demonstrates that phase defects with topological charge \pm 1 occur at the points of change of chirality on the wall. This structure for transverse Bloch domain walls is observed for a wide range of parameters. A typical expanded vision of the amplitude of the field

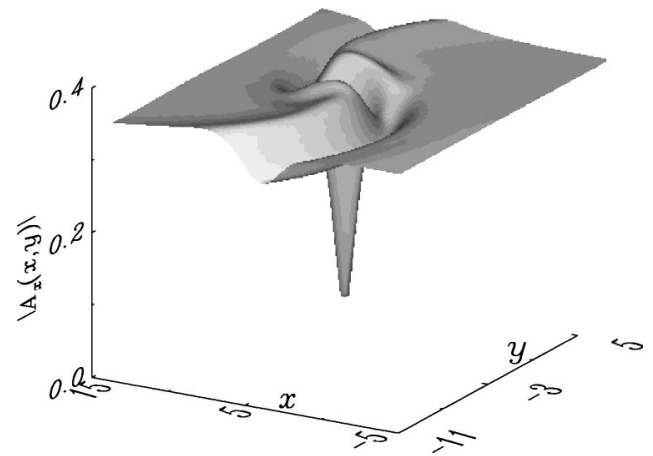

FIG. 9. Amplitude of the $A_{x}$ field as a function of the transverse coordinates $(x, y)$ in the vicinity of a defect. The parameters are the same as in Fig. 3, except $\gamma_{x}=\gamma_{x}^{\prime}=1, c_{x}=0.02(1+i / 2), c_{y}=0.02$.

in the vicinity of a defect is shown in Fig. 9. The corresponding snapshot for the field $A_{y}$ shows the same structure with walls and defects at the same positions as for $A_{x}$.

There are different effects determining the dynamics of BW's in 2D. A first mechanism is the one found in 1D, related to detuning and damping coefficients. However, in $2 \mathrm{D}$ the dynamics is also influenced by the curvature of the walls and the presence of field defects. In what follows two main regimes are identified. In the first regime, called the regime of domain growth, curvature effects dominate. This leads to the complete disappearance of all fronts, all defects, and all domains except one, the final state being a homogeneous solution. The second regime is characterized by a persistent dynamics in which defects are deeply stable objects, while domains and walls are continuously generated and annihilated.

\section{A. Regime of domain growth}

For $\gamma_{x} \Delta_{x}=\gamma_{y} \Delta_{y}$, flat 2D BW's are stable structures. This corresponds to the fact that $1 \mathrm{D}$ walls, for the same values of the parameters, do not move. The transient dynamics is then an ordering process mainly controlled by the curvature effects in which the walls evolve by reducing curvature. This leads to the growth of one of the two equivalent solutions at the expense of the other and the annihilation of all the defects. This process is shown in Fig. 10, where snapshots at different times are shown. The images of the upper row show the intensity of the polarization component $A_{x}$, while in the pictures of the lower row the real part of the same field is presented. Slowly all the defects and walls disappear and the final state is a homogeneous phase-locked solution.

In this regime the normal velocity of the fronts is determined by the local curvature of the wall. This is demonstrated by Fig. 11 where the evolution of the square radius of a domain surrounded by a circular BW has been determined. The result is a growth law $R(t) \simeq t^{1 / 2}$ characteristic of curvature driven domain growth [26]. Similar structures, annihilation dynamics, and dynamical exponents for the growth law of BW domains have been reported in the description of the ordering process of a nonconserved anisotropic $X Y$ spin system in 2D [38]. 


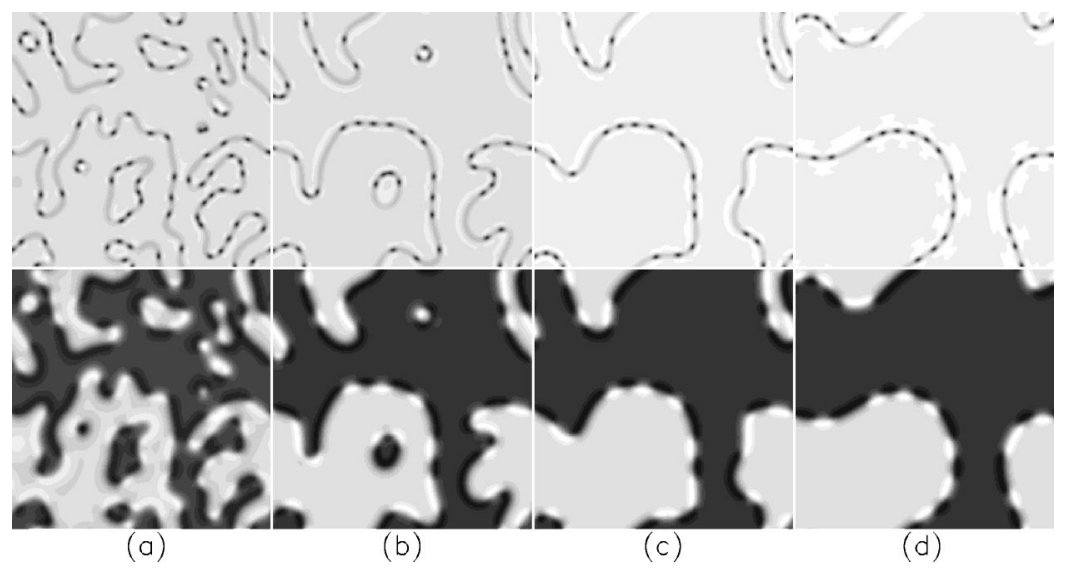

FIG. 10. The domain growth regime $\left(\gamma_{x} \Delta_{x}\right.$ $=\gamma_{y} \Delta_{y}$ ) is presented by means of snapshots at different times: (a) $t=200$; (b) $t=600$; (c) $t$ $=1000$; (d) $t=2000$. The upper row shows the evolution of the intensity of $A_{x}(x, y, t)$ while in the lower row the real part of the same field is shown. The initial condition is random and the parameters are the same as in Fig. 3, except $\Delta_{y}$ $=0.01002, \gamma_{1}=1.002, c_{x}=c_{y}=c_{x}^{\prime}=c_{y}^{\prime}=0.02(1$ $+i)$.

\section{B. Regime of persistent dynamics}

More unusual of our system is the regime found for $\gamma_{x} \Delta_{x} \neq \gamma_{y} \Delta_{y}$. Let us recall that in this regime $1 \mathrm{D}$ optical BW's of different chirality move in opposite directions while IW's (characterized by a point of zero amplitude) do not move. The dynamics in 2D is reminiscent of this behavior; in fact the defects are notably stable like the corresponding 1D IW's, while BW's of different chirality move in opposite directions. The combination of these two effects is that BW's spiral around the defects; this phenomenon was also observed for BW's in other physical systems [39]. The spiraling dynamics of an isolated defect is shown in Fig. 12 obtained with a flat-top profile for the pump beam. The stability of the defects with these physical boundary conditions is remarkable. This is an important result because stabilization of optical vortices has always been critical in lasers and nonlinear optical systems [40]. When many of these defects arise spontaneously from random initial conditions around the trivial unstable solution, the system becomes trapped in a complicated state of persistent dynamics in which a homogeneous state is never reached. An understanding of such persistent dynamics is easier for particular initial conditions. In the example shown in Fig. 13 the initial condition is a flat BW with equally spaced defects along the wall. This gives rise to segments of different chirality along the wall that start moving in opposite directions. The net result, after collision of spiraling BW's of the same chirality, is the periodic emis-

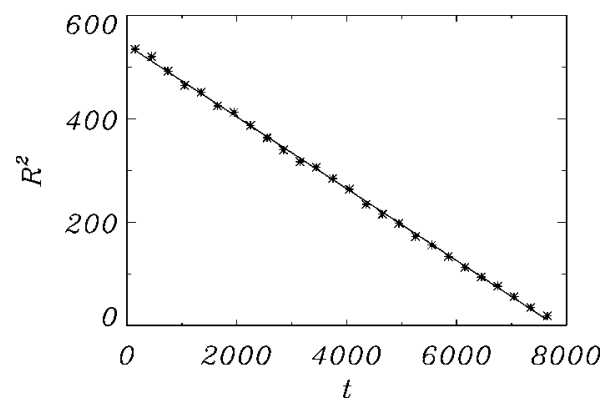

FIG. 11. The asterisks show the time evolution of the square radius $R^{2}$ of a circular BW domain, in the growth domain regime, as they result from the numerical solution. The solid line is a linear interpolation of numerical data. Parameters are the same as in Fig. 10 , except $c_{x}^{\prime}=c_{y}^{\prime}=0.01(1+i)$ and $c_{x}=c_{y}=0.02$. sion, in each direction, of BW's of alternating chirality. The defects remain stable and walls are regenerated by the spiraling process. The dynamical process that we have just described becomes fuzzy close to the Ising-Bloch transition. In this regime of parameters the amplitude of the field becomes very small at the core of the wall and the clear distinction between point defects and wall segments of different chirality is lost.

\section{2D ISING WALLS}

Beyond the transition from BW's to IW's the latter appear spontaneously separating spatial domains of the + and solutions, but their dynamics and the asymptotic state of the system still depend strongly on the strength of the direct polarization coupling. As in the case of BW's, we find for IW's two main regimes: a regime of domain growth and one of labyrinthine pattern formation.

For coupling values close to the Bloch-Ising transition, a flat Ising wall is stable. The transient dynamics is then controlled by the curvature of the walls. Curvature reduction leads to domain growth much in the same way as we already described for BW's in the corresponding regime. In Fig. 14(a) typical time series of snapshots of the transverse $A_{x}$ field is shown. The final asymptotic state is homogeneous.

(a)
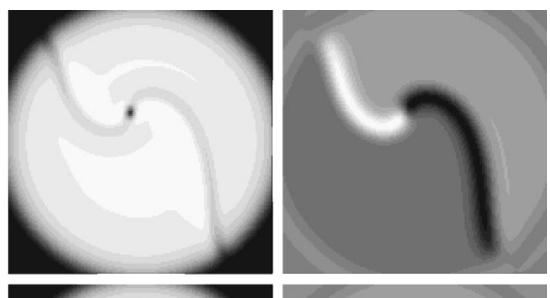

(b)
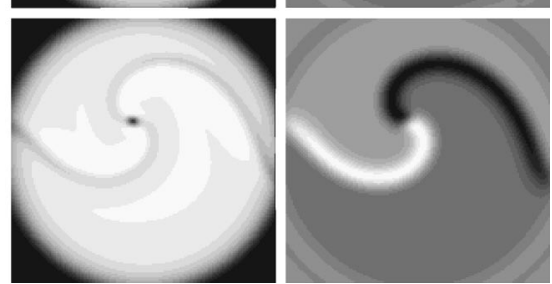

FIG. 12. Time evolution of one spiral of BW's around a defect. In the left column the amplitude of $A_{x}(x, y, t)$ is represented while in the right column the real part of the same field is shown. (a) $t$ $=560$; (b) $t=1120$. The parameters are the same as in Fig. 9 . 
(a)

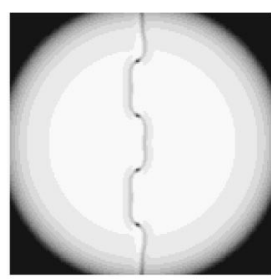

(b)

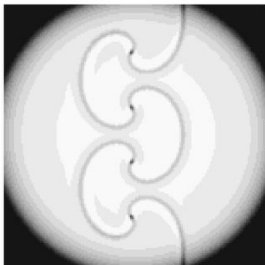

(c)

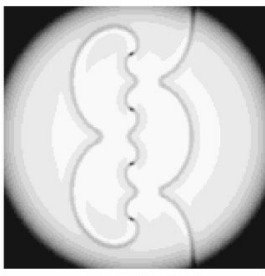

(d)

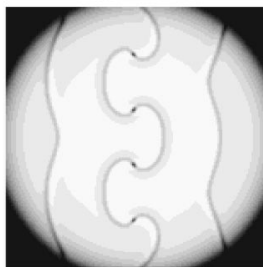

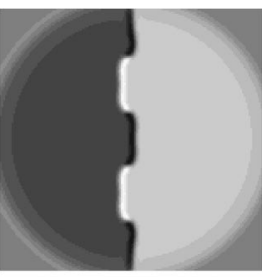
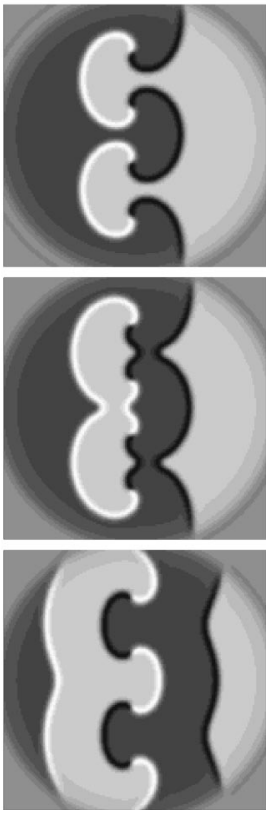

FIG. 13. Time evolution of a BW, spiraling around an array of defects imposed as an initial condition: (a) $t=0$; (b) $t=1000$; (c) $t=1150$; (d) $t=1550$. In the left column the amplitude of $A_{x}(x, y, t)$ is represented while in the right column the real part of the same field is shown. The parameters are the same as in Fig. 7 except $\Delta_{x}=0.01, \Delta_{y}=0.03, c_{x}=c_{y}=0.025$, and $c_{x}^{\prime}=c_{y}^{\prime}=0.01$.

Similar dynamics has already been reported in a type-I DOPO above threshold $[8,11,20]$, the only difference being the vectorial character of the field in this case.

Far from the Bloch-Ising transition and moving deeper into the locking regime transverse labyrinthine patterns are spontaneously formed in the system starting from a random perturbation of the trivial unstable solution [26,27]. Snapshots of the evolution of a pattern of this type are shown for intensity and real part in Fig. 15. Note that the time evolution

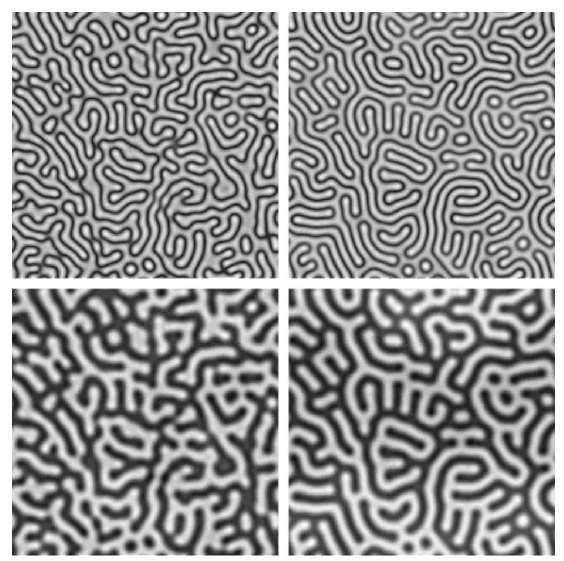

(a)

(b)

FIG. 15. Formation of a labyrinthine pattern in the regime for which IW's are stable but their curvature is modulationally unstable: the upper row shows the intensity pattern and the lower row $\operatorname{Re}\left(A_{x}\right)$ for (a) $t=75$; (b) $t=1500$. The initial condition is random and the values of the parameters are the same as in Fig. 14, except $\Delta_{y}=0.01001, c_{x}=c_{y}=c_{x}^{\prime}=c_{y}^{\prime}=0.3 i$.

of labyrinthine patterns is very slow. The creation of a labyrinthine pattern stems from the fact that, in this regime of parameters, flat Ising walls (i.e., with no curvature) are modulationally unstable. Roughly speaking the finger growth is associated with a band of modulational frequencies of the front curvature that tend to increase their curvature. This is reminiscent of what has been reported for intracavity second harmonic generation [23] and vectorial Kerr resonators [26] . In order to illustrate the modulational instability, the evolution of an initially perturbed Ising flat wall is shown in Fig. 16.

\section{CONCLUSION}

In conclusion we have demonstrated that Bloch walls can be found in nonlinear optical systems, in particular in type-II optical parametric oscillators. They appear when there exists a small birefringence and/or dichroism of the cavity along axes that do not coincide with those of the nonlinear crystal. These effects introduce a linear coupling between the signal and the idler which causes self-phase-locking of the two

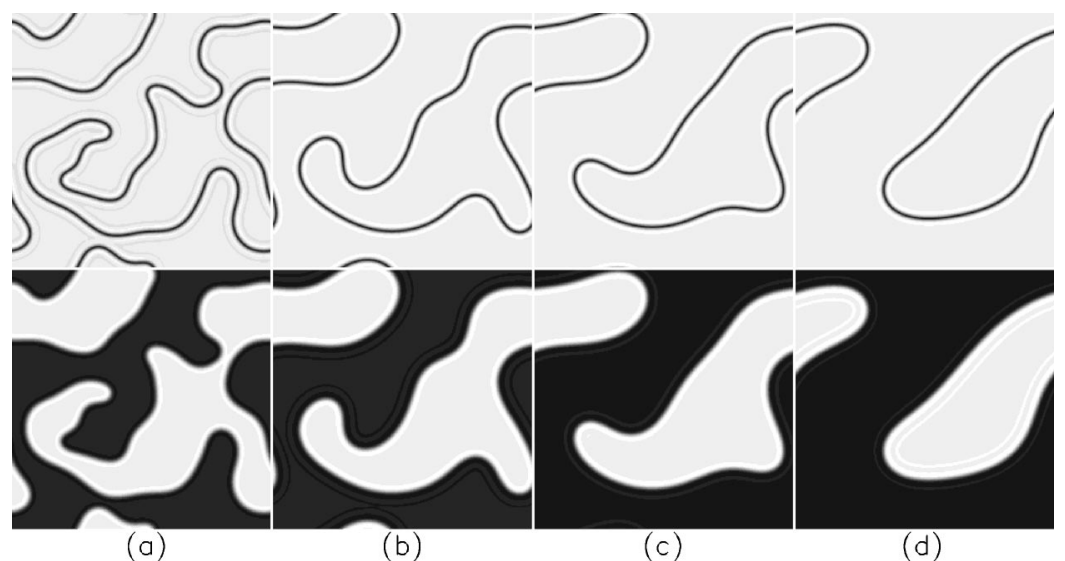

(a) (b) (c) (d)
FIG. 14. Snapshot at different times, observed in the coarsening regime: (a) $t=300$; (b) $t=900$; (c) $t=1900$; (d) $t=3000$. The upper row presents the intensity pattern and the lower row $\operatorname{Re}\left(A_{x}\right)$. The initial condition is random and the values of the parameters are the same as in Fig. 3, except $c_{x}=c_{y}=0.09$. 


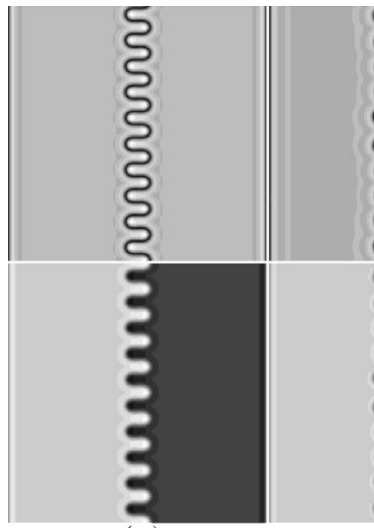

(a)

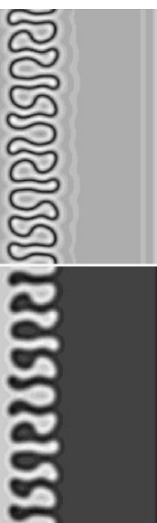

(b)

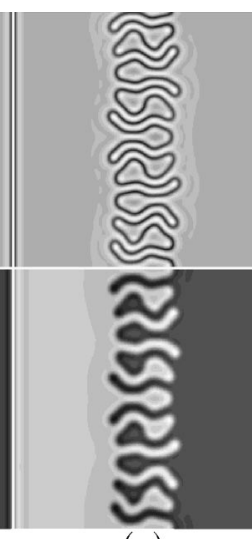

(c)

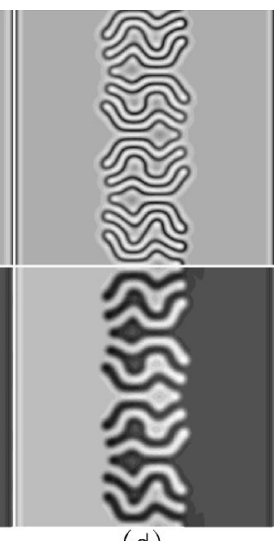

(d)
FIG. 16. Modulational instability of an initially flat IW: the upper row shows $\left|A_{x}(x, y, t)\right|^{2}$ and the lower row the real part of the same field. In this regime 1D Ising fronts are stable. The values of the parameters are the same as in Fig. 15. fields. There exist two possible steady state solutions, characterized by a phase shift of $\pi \mathrm{rad}$ of both polarization components, and thus different domains spontaneously form in which one or the other solution is selected. The separating walls can be of either the Bloch or the Ising type depending on the strength of the coupling coefficient. For small values Bloch walls are stable and appear spontaneously above a predicted threshold out of a random perturbation of the trivial steady state. Bloch walls have been characterized in both one and two dimensions. In one dimension a physical interpretation of Bloch walls is given in terms of polarization variations that connect two homogeneous states that represent the same state of polarization. The chirality is instead related to the ellipticity variations. Multiple hump Ising walls have also been found starting from particular initial conditions. In two dimensions, Bloch walls can possibly have wall sections of different chirality, i.e., where the phase rotates in two possible ways, clockwise or counterclockwise in the complex plane. Where the chirality changes sign the phase has defects, where the field amplitude is zero, and the wall degenerates into an Ising one. Two dynamical regimes, which depend on the decay rates and the detunings, are found: in the first one, the wall dynamics is dominated by the curvature and a final homogeneous state is reached; in the second regime, the walls spiral around stable defects and persistent creation and annihilation of fronts is observed. The transition from Bloch to Ising walls has also been observed when the linear coupling strength is increased; the transition is characterized by larger and larger variations of the amplitude of the field close to the core of the wall and the fact that the walls stop moving. Ising wall dynamics has also been considered, in particular, the curvature modulation instability, which leads to the creation of a labyrinthine pattern.

\section{ACKNOWLEDGMENTS}

This work was partially supported by the Spanish MCyT Project No. BFM2000-1108 and by the European Commission project QSTRUCT (FMRX-CT96-0077). The authors acknowledge clarifying discussions with G.-L. Oppo.

\section{APPENDIX}

In this appendix the derivation of Eqs. (1) is presented. The main difference with respect to previous derivations of mean field equations for OPO's is the inclusion of the effects of dichroic and birefringent mirrors. In order to simplify the model, let us assume that only one out of the four mirrors of the ring cavity is birefringent and dichroic. This means that, in a proper system of orthogonal axes, the matrix $M$, which represents the transformation of two orthogonally polarized components of a beam, in the Jones formalism [32] is

$M=\left(\begin{array}{cc}r_{1} e^{-i \psi_{1}} & 0 \\ 0 & r_{2} e^{-i \psi_{2}}\end{array}\right)=r e^{-i \sigma}\left(\begin{array}{cc}(1+p) e^{i \delta} & 0 \\ 0 & (1-p) e^{-i \delta}\end{array}\right)$

where $\quad r=\left(r_{1}+r_{2}\right) / 2, p=\left(r_{1}-r_{2}\right) /(2 r), \sigma=-\left(\psi_{1}+\psi_{2}\right) / 2$, and $\delta=\left(\psi_{2}-\psi_{1}\right) / 2$. The dichroism, i.e., the different reflectivity of the mirror for different polarizations $\left(r_{1} \neq r_{2}\right)$, implies that $p \neq 0$, while the birefringence is represented by the fact that $\psi_{1} \neq \psi_{2}$, i.e., the phases of the reflected components undergo a different change $(\delta \neq 0)$.

A first important remark to make is that the mirror anisotropy axes might not coincide with the axes of the nonlinear crystal, which is also birefringent in order to realize the phase matching between the pump and the generated fields. In other words the anisotropic crystal has its own preferred polarization axes that can be rotated by an angle $\phi$ with respect to the mirror principal axes (those for which the mirror matrix is diagonal). Therefore, when passing from the propagation in the cavity axes reference frame to that in the crystal a rotation, represented by the matrix

$$
R=\left(\begin{array}{cc}
\cos \phi & -\sin \phi \\
\sin \phi & \cos \phi
\end{array}\right),
$$

has to be applied. At the end of the propagation in the nonlinear medium an inverse transformation $\left(R^{-1}\right)$ is needed to restore the reference frame of the cavity axes.

The generic $n+1$ round trip in the cavity for the signal and idler vector fields of the previous step $n\left(\vec{E}_{n}\right)$ is then represented by the following transformation (all mirrors except the last one are perfectly reflective, i.e., their matrices are all equal to the identity matrix):

$$
\vec{E}_{n+1}=M R^{-1} f\left(R \vec{E}_{n}\right)
$$


where $f(\cdot)$ is the result of the propagation inside the nonlinear medium. A similar formula can be written also for the pump vector field $\vec{F}_{n}$ which, in principle, has both polarization components. Usually one component does not participate in the nonlinear dynamics (it is not phase matched) and it is neglected a priori. In this case, due to polarization coupling, it is included in the model although the final result is that, under not very restrictive hypotheses, its effects can be neglected.

The output of the function $f(\cdot)$ is the vector field as it results after the integration of a set of nonlinearly coupled propagation equations, i.e., it involves products of $\vec{E}_{n}$ and $\vec{F}_{n}$. Hereafter only the signal and idler vector field $\vec{E}_{n}$ equations will be considered; similar calculations can be repeated for the pump components.

Let us remark that the optical carrier frequency of each component has already been removed (i.e., envelope equations [36] are searched for) and that the carrier frequencies of all waves are determined by three conditions: phase matching and energy conservation of the nonlinear interaction and the condition of resonance due to the cavity. It has been demonstrated [35] that, for a type-II OPO, there are several signal-idler pairs of oscillation frequencies that can satisfy these conditions. Among these solutions there is also the case of quasi, or totally, frequency degenerate signal and idler. This is also verified experimentally by the fact that type-II OPO's, unlike type-I OPO's, can be smoothly tuned through frequency degeneracy $[16,36]$. Let us remark, finally, that for type-II OPO's signal and idler are always polarization nondegenerate.

The boundary condition to impose on Eq. (A3) for steady state operation of the OPO is that the round trip transformation coincides with the identity, i.e., $\vec{E}_{n+1}=\vec{E}_{n}$. Let us define $\vec{A}(L)=f\left(R \vec{E}_{n}\right)$, the vector field at the output of a crystal of length $L$ (which is also the cavity length for the completely filled cavity) and $\vec{A}(0)=R \vec{E}_{n}=R \vec{E}_{n+1}$, the field at the crystal input; by multiplying the left and right hand sides of Eq. (A3) by $R$ (on the left) and substituting previous definitions the following equation is found:

$$
\vec{A}(0)=R M R^{-1} \vec{A}(L) .
$$

Let us now set $\vec{A}^{\prime}(z)=H(z) \vec{A}(z)$ such that

$$
\vec{A}^{\prime}(0)=\vec{A}(0), \quad \vec{A}^{\prime}(L)=\vec{A}^{\prime}(0)
$$

the second condition imposing the periodicity after a round trip. The general form of the matrix $H(z)$ satisfying Eq. (A5) is

$$
H(z)=\left(\begin{array}{cc}
e^{h_{x x} z} & e^{h_{x y} z}-e^{-h_{x y} z} \\
e^{h_{y x} z}-e^{-h_{y x} z} & e^{h_{y y} z}
\end{array}\right) .
$$

This matrix extends the scalar transformation used by Lugiato and Oldano in their original paper [41], devoted to the study of stationary spatial patterns in optical systems with two-level atoms, to a vectorial case. It is easy to verify that
$H(0)$ is the identity matrix while $H(L)=R M R^{-1}$ and thus all the elements of the matrix $h_{i j}$ can be explicitly calculated:

$$
\begin{aligned}
h_{x x}= & \frac{1}{L}\{\ln (r)-i \sigma+\ln [(1+p \cos 2 \phi) \cos \delta \\
& +i(p+\cos 2 \phi) \sin \delta]\}, \\
h_{y y}= & \frac{1}{L}\{\ln (r)-i \sigma+\ln [(1-p \cos 2 \phi) \cos \delta \\
& +i(p-\cos 2 \phi) \sin \delta]\}, \\
h_{x y}=h_{y x} & =\frac{1}{L}\{\ln [(p \cos \delta+i \sin \delta) \sin 2 \phi \\
+ & \left.\left.\sqrt{(p \cos \delta+i \sin \delta)^{2} \sin ^{2} 2 \phi+4}\right]-\ln (2)\right\} .
\end{aligned}
$$

The evolution of the field in the crystal is governed by equations of the type

$$
\partial_{z} \vec{A}=\mathcal{L}(\vec{A})+\mathcal{N}(\vec{A}, \vec{B})
$$

where $\vec{B}=\left[B_{x}, B_{y}\right]=R \vec{F}_{n}$ represents the pump vector field. The linear term is

$$
\mathcal{L}(\vec{A})=\left(\begin{array}{cc}
\frac{i}{2 k_{x}} \nabla^{2}-\frac{1}{v_{x}} \partial_{t} & 0 \\
0 & \frac{i}{2 k_{y}} \nabla^{2}-\frac{1}{v_{y}} \partial_{t}
\end{array}\right) \vec{A}
$$

and includes the diffraction $\left(k_{x, y}\right.$ are the longitudinal wave vectors of signal and idler, $\nabla^{2}$ is the spatial transverse Laplacian operator) and the phase velocity mismatch $\left(v_{x, y}\right.$ are the phase velocities, respectively, of signal and idler, $\partial_{t}$ is the differential operator with respect to time). The nonlinear operator is

$$
\mathcal{N}(\vec{A}, \vec{B})=i K B_{x}\left(\begin{array}{ll}
0 & 1 \\
1 & 0
\end{array}\right) \vec{A}^{*}
$$

where $K$ is the nonlinear coefficient. Since $\vec{A}=H^{-1} \vec{A}^{\prime}$ the evolution of the field $\vec{A}^{\prime}$ in the crystal can be determined from

$$
\partial_{z} \vec{A}=\left(\partial_{z} H^{-1}\right) \vec{A}^{\prime}+H^{-1} \partial_{z} \vec{A}^{\prime}
$$

which finally yields

$$
\partial_{z} \vec{A}^{\prime}+H\left(\partial_{z} H^{-1}\right) \vec{A}^{\prime}=H \mathcal{L}\left(H^{-1} \vec{A}^{\prime}\right)+H \mathcal{N}\left(H^{-1} \vec{A}^{\prime}, \vec{B}\right) .
$$

The matrix $H$ is known and so all the terms of the last equation can be explicitly calculated; in particular, by following the guidelines of Ref. [41], if $p$ and $\delta$ are small parameters (of the order of the transmittivity $T=1-r$ of the mirror) one can calculate a set of first order (in T) equations by expanding all coefficients up to this order. After long but straight- 
forward calculations, the final result is a set of coupled equations for the vector $\vec{A}^{\prime}=\left[A_{x}, A_{y}\right]^{T}$, that still contains both $\partial_{z}$ and $\partial_{t}$ operators, but includes the boundary conditions. It is worth writing these equation because the coefficient of each term appears explicitly written in terms of physical parameters:

$$
\begin{aligned}
\partial_{t} A_{x}+v_{x} \partial_{z} A_{x}= & h_{x x} v_{x} A_{x}+\frac{v_{x}}{L}(p+i \delta) \sin 2 \phi A_{y}+i \frac{v_{x}}{2 k_{x}} \nabla^{2} A_{x} \\
& +i v_{x} K A_{y}^{*} B_{x}, \\
\partial_{t} A_{y}+v_{y} \partial_{z} A_{y}= & h_{y y} v_{y} A_{y}+\frac{v_{y}}{L}(p+i \delta) \sin 2 \phi A_{x}+i \frac{v_{y}}{2 k_{y}} \nabla^{2} A_{y} \\
& +i v_{y} K A_{x}^{*} B_{x} .
\end{aligned}
$$

By exploiting the single longitudinal mode approximation, which is quite a good one for continuous wave OPO's, the longitudinal spatial dependence can be finally removed and the equations describing the time evolution are exactly Eqs. (1). The coefficients of Eqs. (1) can be found from Eqs. (A13) once the expressions (A7) are substituted. They read

$$
\begin{gathered}
\gamma_{x, y}=\frac{v_{x, y}(T \pm p \cos 2 \phi)}{L}, \\
\Delta_{x, y}=\frac{v_{x, y}(\sigma \pm \delta \cos 2 \phi)}{T \pm p \cos 2 \phi},
\end{gathered}
$$

$$
\begin{aligned}
\alpha_{x, y} & =\frac{L}{2 k_{x, y}(T \pm p \cos 2 \phi)}, \\
c_{x, y} & =\frac{p+i \delta}{T \pm p \cos 2 \phi} \sin 2 \phi,
\end{aligned}
$$

where the plus (minus) sign applies for the $x$-polarized ( $y$-polarized) component. Note that all coefficients can be different because of the crystal birefringence $\left(v_{x} \neq v_{y}\right)$, and the cavity birefringence and/or dichroism $(p \neq 0, \delta \neq 0)$. Finally the nonlinear coefficient is defined as

$$
K_{0}=\frac{K L}{T} .
$$

Actually it is also slightly different for the two polarization, but this difference has been neglected because it is only due to the mirror dichroism; all the other coefficients have larger differences because two effects (crystal and cavity birefringence) contribute.

Regarding the coupling coefficients some special cases are worth remarking in relation to what is discussed in other sections of this paper. For example, when $\cos 2 \phi=0$, the special condition $c_{x}=c_{y}$ (complex) is obtained; in this case $\phi= \pm \pi / 4, \pm 3 \pi / 4, \sin 2 \phi= \pm 1$, and therefore the linear coupling among polarization is maximized in modulus. Two other special cases are the purely birefringent mirror $p=0$, for which $c_{x}=c_{y}$ are purely imaginary, and the purely dichroic mirror which yields $\delta=0$ and thus purely real $c_{x, y}$.
[1] J. Opt. Soc. Am. B 16 (1999), special issue.

[2] M. D. Reid and P. Drummond, Phys. Rev. Lett. 60, 2731 (1988); J. Mertz, T. Debuisschert, A. Heidmann, C. Fabre, and E. Giacobino, Opt. Lett. 16, 1234 (1991); P. G. Kwiat, K. Mattle, H. Weinfurter, A. Zeilinger, A. V. Sergienko, and Y. Shih, Phys. Rev. Lett. 75, 4337 (1995).

[3] L. Lugiato, A. Gatti, and H. Wiedemann, in Quantum Fluctuations and Nonlinear Optical Patterns, edited by S. Reynaud, E. Giacobino, and J. Zinn-Justin, Proceedings of the Les Houches Summer School of Theoretical Physics, Session LXIII, 1995 (Elsevier, Amsterdam, 1997).

[4] J. Opt. B: Quantum Semiclass. Opt. 1 (1999), special issue.

[5] G.-L. Oppo, M. Brambilla, and L. A. Lugiato, Phys. Rev. A 49, 2028 (1994).

[6] G. J. de Valcarcel, K. Staliunas, E. Roldan, and V. J. SanchezMorcillo, Phys. Rev. A 54, 1609 (1996).

[7] M. Vaupel, A. Matre, and C. Fabre, Phys. Rev. Lett. 83, 5278 (1999).

[8] G.-L. Oppo, A. J. Scroggie, and W. J. Firth, Phys. Rev. E (to be published).

[9] M. Le Berre, D. Leduc, E. Ressayre, and A. Tallet, J. Opt. B: Quantum Semiclass. Opt. 1, 153 (1999); M. Tlidi, M. Le Berre, E. Ressayre, A. Tallet, and L. Di Menza, Phys. Rev. A 61, 043806 (2000).

[10] G. Izús, M. Santagiustina, and M. San Miguel, Opt. Lett. 25, 1454 (2000).
[11] S. Trillo, M. Haelterman, and A. Sheppard, Opt. Lett. 22, 970 (1997).

[12] S. Longhi, Phys. Scr. 56, 611 (1997).

[13] M. Santagiustina, P. Colet, M. San Miguel, and D. Walgraef, Opt. Lett. 23, 1167 (1998).

[14] K. Staliunas and V. Sánchez-Morcillo, Phys. Rev. A 57, 1454 (1998).

[15] G. L. Oppo, A. J. Scroggie, and W. J. Firth, J. Opt. B: Quantum Semiclass. Opt. 1, 133 (1999).

[16] E. J. Mason and N. C. Wong, Opt. Lett. 23, 1733 (1998).

[17] C. Fabre, E. Mason, and N. Wong, Opt. Commun. 170, 299 (1999).

[18] G. Izús, M. Santagiustina, M. San Miguel, and P. Colet, J. Opt. Soc. Am. B 16, 1592 (1999).

[19] N. Kutz, T. Ernaux, S. Trillo, and M. Haelterman, J. Opt. Soc. Am. B 16, 1936 (1999).

[20] G.-L. Oppo, A. J. Scroggie, and W. J. Firth (unpublished).

[21] P. Coullet, J. Lega, B. Houchmanzadeh, and J. Lajzerowicz, Phys. Rev. Lett. 65, 1352 (1990).

[22] E. G. Westhoff, V. Kneisel, Y. A. Logvin, T. Ackermann, and W. Lange, J. Opt. B: Quantum Semiclass. Opt. 2, 386 (2000).

[23] U. Peschel, D. Michaelis, C. Etrich, and F. Lederer, Phys. Rev. E 58, R2745 (1998).

[24] M. Tlidi, P. Mandel, and R. Lefever, Phys. Rev. Lett. 81, 979 (1998).

[25] K. Staliunas and V. Sánchez-Morcillo, Phys. Lett. A 241, 28 (1998). 
[26] R. Gallego, M. San Miguel, and R. Toral, Phys. Rev. E 61, 2241 (2000).

[27] V. Taranenko, K. Staliunas, and C. Weiss, Phys. Rev. Lett. 81, 2236 (1998).

[28] N. N. Rozanov, Prog. Opt. 35, 1 (1996).

[29] D. Michaelis, U. Peschel, F. Lederer, D. V. Skryabin, and W. Firth, Phys. Rev. E 63, 066602 (2001); G. de Valcarcel and K. Staliunas (unpublished).

[30] H. J. Kimble, in Quantum Fluctuations in Quantum OpticsSqueezing and Related Phenomena, edited by J. Dalibard, J. M. Raimond, and J. Zinn-Justin (Elsevier, Amsterdam, 1992).

[31] D. Lee and N. Wong, Appl. Phys. B: Lasers Opt. 66, 133 (1998).

[32] B. E. A. Saleh and M. C. Teich, The Fundamentals of Photonics (Wiley, New York, 1991), Chap. 6.

[33] J. Falk, IEEE J. Quantum Electron. QE-7, 230 (1971).

[34] R. Eckardt, C. D. Nabors, W. J. Kozlowsky, and R. L. Byer, J. Opt. Soc. Am. B 8, 646 (1991).

[35] T. Debuisschert, A. Sizmann, E. Giacobino, and C. Fabre, J.
Opt. Soc. Am. B 10, 1668 (1993).

[36] J.-Y. Zhang, J. Y. Huang, and Y. R. Shen, Laser Sci. Technol. 19, 1 (1995); C. L. Tang and L. K. Cheng, ibid. 20, 1 (1995).

[37] Equations (1) have been integrated using the algorithm described in Ref. [42]. In 1D we take a grid of 2048 samples, with periodic boundary conditions. For all cases the grid space was $\Delta x=0.078125$ and the integration step was $\Delta t=0.001$. For the $2 \mathrm{D}$ case we use a grid of $256 \times 256$ samples with grid space $\Delta x=\Delta y=0.3125$ and time step $\Delta t=0.02$. In some cases we use, instead of periodic boundary conditions, a flat-top super-Gaussian pump beam $E_{0}(x, y)$.

[38] H. Tutu, Phys. Rev. E 56, 5036 (1997).

[39] T. Frisch, S. Rica, P. Coullet, and J. M. Gilli, Phys. Rev. Lett. 72, 1471 (1994).

[40] C. O. Weiss, M. Vaupel, K. Staliunas, G. Slekys, and V. B. Taranenko, Appl. Phys. B: Lasers Opt. 68, 151 (1999).

[41] L. A. Lugiato and C. Oldano, Phys. Rev. A 37, 3896 (1988).

[42] R. Montagne, E. Hernández-García, A. Amengual, and M. San Miguel, Phys. Rev. E 56, 151 (1997). 